\title{
Drei schweizerische Erstleistungen in der Frühgeschichte des Panoramas
}

Seit der vorjährigen Ausstellung von Ansichten und Panoramen HANS CONRAD ESCHERS VON DER LINTH (1767-1823) in der Zentralbibliothek Zürich ${ }^{1)}$ ist der Beitrag des bedeutenden Zürchers zur Genesis des Panoramas der schweizerischen Öffentlichkeit bekannt: Der Staatsmann und Naturforscher, Journalist, Wirtschafts- und Sozialpolitiker, treibende Kraft und Leiter des ersten nationalen Aufbauwerkes der Schweiz und Retter von 16000 Bewohnern der Linthebene aus Sumpffieber und Elend - dieser Mann, ein unermüdlicher Gebirgswanderer und -zeichner, gesellt sich als dritter zu den beiden bisher bekannten Erfindern des modernen Panoramas. Die nähere Untersuchung brachte Erkenntnisse, die hier erstmals in knapper Übersicht publiziert werden. Als Ausgangspunkt ist zunächst die Situation und Eschers Leistung zusammenzufassen:

Im Jahre 1787 präsentierte der Porträtmaler ROBERT BARKER dem Direktor der Londoner Kunstakademie Sir Joshua Reynolds eine Ansicht der Stadt Edinburg auf halbrund aufgestellter ungerahmter Leinwand. Das seltsame Bild fand keine Anerkennung; dessenungeachtet lie $\beta$ Barker seine Erfindung patentieren. Erfolglos blieb auch ein weiterer Versuch Barkers, Edinburg in einem geschlossenen Rundbild darzustellen. Eine halbkreisförmige Ansicht von London in ungewöhnlich großen Dimensionen fand ersten Anklang, aber erst ein riesiges Rundpanorama mit der Darstellung der russischen Kriegsflotte vor Spithead, in einer eigens dafür erbauten Rotunde von $30 \mathrm{~m}$ Durchmesser aufgespannt und 1794 der Öffentlichkeit zugänglich gemacht, brachte Barker den ersehnten Publikums- und Kassenerfolg. Das «Panorama», wie der Erfinder seine Schöpfung benannte ${ }^{2)}$, wurde populär und alsbald Mode.

In den neunziger Jahren des 18. Jahrhunders entwickelte auch der Danziger Architekturzeichner und Bühnenbildner JOHANN ADAM BREYSIG eine theoretische Konzeption des Panoramas, die er 17891801 publizierte $^{3)}$. Zudem hatte er nach eigenem Bericht schon 1792 eine Rundansicht von Rom skizziert, die er jedoch erst im Jahre 1800 zum ersten Berliner Panoramagemälde ausarbeiten konnte ${ }^{4}$. Barker und Breysig galten bisher als die Erfinder des Panoramas.

H. C. ESCHERS 3,5 m lange Zirkularaussicht vom Fieudo im Gotthardmassiv entstand jedoch 1792, zwei Jahre bevor der Gedanke des Panoramas über- haupt Fuß faßte. Escher ist somit - unabhängig von den beiden Vorgenannten - einer der Erfinder des modernen Panoramas. Dafür gibt es ein Selbstzeugnis. In einem Bericht für «Leonhards Taschenbuch der Mineralogie» ${ }^{5)}$ schreibt er im Dezember 1811 u. a.: "So z. B. zeichnete ich schon im Jahre 1792, als man noch an keine Panoramen dachte, auf der Spizze des Fieudo am Gottharde eine vollständige Zirkularaussicht: noch war mir das Streichen der Gebirgsketten unbekannt, und über die Schichtensenkung hatte ich sehr unbestimmte Begriffe. Erst mehrere Jahre nachher entdeckte ich am Gottharde den fächerförmigen Stand der Schichten: aber die Ausdehnung dieser Schichtung war mir noch unbekannt: ich zog mein Fieudo-Panorama hervor, spannte dasselbe in einen Reif, orientierte es, und fand den fächerförmigen Schichtenstand in seinen Profilansichten so auffallend darin aufgezeichnet, $\mathrm{da} ß$ mein Überblick über die Schichtung des Gotthards sehr erweitert wurde." Die weiteren Zeilen des Berichts lassen erkennen, wie stark dabei seine emotionellen Motive waren: «... neben diesen wissenschaftlichen Resultaten, welche solche richtig gezeichnete Gebirgsansichten oft lange nachdem sie aufgenommen wurden, zu liefern im Stande sind, welch ein Genuß kann ich mir nicht verschaffen, wenn ich, in Zeitverhältnissen, die keine Alpenwanderungen gestatten, meine Zirkularaussichten... aufspanne, und jeden einzelnen Gebirgsstock, den man dort sieht, wieder betrachten, oder den ganzen Zusammenhang derselben übersehen kann? Auch die lebhafteste Einbildungskraft ist diesen Genuß nicht zu verschaffen vermögend...» Einschränkend fügt Escher jedoch hinzu: «Bei diesen geognostischen Zeichnungen muß aber die Kunst etwas zurückgesetzt werden, diese erfordert Luftperspektiv, harmonische Beleuchtung, verwirft Härte der Umrisse und der umständlicheren Unebenheiten; gerade jenes hingegen bedarf der Geognost nicht, und dieses ist ihm hingegen sehr wesentlich. Der geognostische Zeichner soll in entfernteren Gebirgsstöcken ... mit dem Fernglase die Linien aufsuchen, welche vielleicht die Schichtenablösung bezeichnen, und ihm einst über die Schichtung von Gebirgsstöcken Auskunft zu geben im Falle sind, die er

Dr. G. Solar, Graphische Sammlung der Zentralbibliothek Zürich, Zähringerplatz 6, 8001 Zürich 


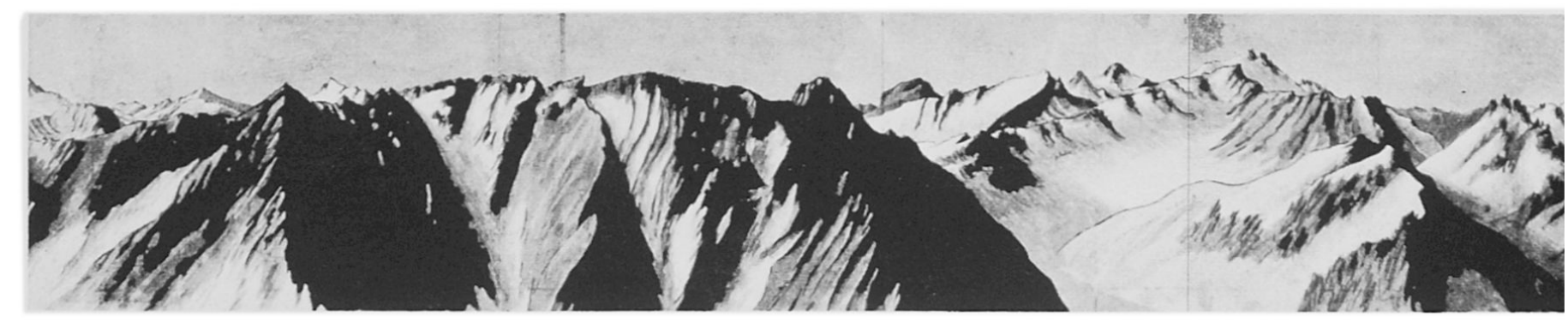

jetzt noch gar nicht kennt: - der Maler hingegen darf oft ganz nahe und deutlich sich darstellende Schichtenprofile kaum bemerken, weil sie Härte bewirken, und schöne Schatten oder Lichtmassen unterbrechen würden.» Ungeachtet der Tatsache, daß Eschers künstlerisches Temperament ihn diese Selbstbeschränkung zuweilen vergessen ließ und er das Streichen der Schichtenprofile gern zur ästhetisch wirkungsvollen Stilisierung steigerte, hier stehen wir vor einer theoretischen Grundlegung der topographischen Gebirgsaufnahme mit ihrer Abgrenzung gegen die Malerei, die Escher weitgehend vom Autor des «Discours sur l'Histoire Naturelle de la Suisse» im 1. Band der Zurlaubenschen "Tableaux topographiques, pittoresques, etc. ... de la Suisse» (Paris 1777-1788), dem französischen Geologen Besson, übernommen hat ${ }^{6}$.

Damit wird klar, daß es Escher um etwas anderes $\mathrm{zu}$ tun ist als Barker und Breysig. Wenn Buddemeier (s. Anm. 2) unter Berufung auf Diderot meint, mit dem Panorama Barkers und Breysigs entständen «zum ersten Mal Abbildungen, die nur das eine Ziel haben, einen Gegenstand so exakt wie möglich wiederzugeben und den Betrachter so vollkommen wie möglich zu täuschen", so trifft auf Escher die erste, nicht aber die zweite Feststellung zu. Escher wollte nicht die «illusion totale», die Nachahmung der Wirklichkeit mit Hilfe der letzten Errungenschaften von Kunst und Technik, sondern die Vermittlung einer durch die Wissenschaft zugänglich gemachten wirklichen Form und Schönheit. Er brauchte für seine Panoramen nicht die Illusion eines Felsgipfels im verdunkelten Raum, etwa wie Barker in der Mitte seiner Rotunde das Verdeck einer Fregatte aufbaute, von dem aus man die russische Flotte vor Spithead liegen sah. Eschers gar nicht als Kunst verstanden sein wollende Gebirgsaufnahmen sprechen uns Heutige aus eben diesem Grund emotionell stärker an als die ungleich ambitiöseren Arbeiten der erwähnten Großpanoramenmaler und ihrer Nachfolger.

$\mathrm{Zu}$ bescheiden, um mit seinen Zeichnungen vor die Öffentlichkeit zu treten, ist Escher als eigentlicher Begründer einer schweizerischen Panoramenzeichnerschule unbekannt geblieben. Seine Nachfolger erreichten schnell eine neue Qualität und Sonderstellung im europäischen Panoramenschaffen, die auf ihre wissenschaftliche, geographisch-topogra- phische Grundhaltung zurückzuführen ist. Sie haben das Panorama zu einem heute noch - wenngleich vor allem in der Photographie und im Film brauchbaren Instrument der Gebirgsdarstellung gemacht ${ }^{7)}$.

Es würde zu weit führen, hier aufzuzeigen, wie Escher zum Gebirgs-und Panoramenzeichner wurde. Angedeutet sei nur, daß er durch Vermittlung seines Zeichenlehrers J. B. BULLINGER, von dem panoramatische Ansichten Zürichs von der Limmat her erhalten sind, sowohl den Sinn der Niederländer für die panoramatische Landschaft, als auch die panoramatische Sicht des Venezianers Canaletto und dessen Arbeitsweise unter Anwendung der camera obscura mitbekam, die sogar auf zwei Panoramen Eschers in der Staffage abgebildet ist. Ausführlicher werden diese Wurzeln seines Schaffens im Begleittext zur Publikation seiner wichtigsten Panoramen behandel $t^{8}$.

So steht H. C. Escher am Ende einer langen Entwicklung. Die Genesis des Panoramas mußte zurückverfolgt werden, wenn Eschers Leistung ganz verständlich sein sollte. Dabei kam ein Fingerzeig aus dem Publikum der Feststellung einer weiteren schweizerischen Erstleistung zugute - ein Besucher der Ausstellung erkannte in Eschers Horizontalpanorama vom Buet-Gipfel die Kopie der Radierung Planche $8 \mathrm{im} \mathrm{1.} \mathrm{Band} \mathrm{der} \mathrm{"Voyages} \mathrm{dans} \mathrm{les} \mathrm{Alpes»}$ von HORACE-BENÉDICT DE SAUSSURE (1740-1799). Der Typ des Horizontalpanoramas, das später häufig für Orientierungstafeln auf Aussichtspunkten verwendet wurde und die topographischen Daten nicht auf einen Zylinder, sondern in eine Kreisfläche projiziert, kommt bei Escher nur dieses einzige Mal vor. Escher lernte das 1779 in Neuchâtel herausgegebene Werk Saussures 1785 während seines Aufenthaltes in Genf kennen ${ }^{9)}$, wo er sich, 18 Jahre alt, zwecks Vervollkommnung seiner Französischkenntnisse aufhielt. Sein Privatlehrer war dort der spätere Freund seines Lebens, J. P. Vaucher, Theologe und Botaniker, mit dem Escher seine ersten Bergwanderungen unternahm, u.a. auch eine Besteigung des Buet ${ }^{10}$. Daß Saussure der Erfinder des Horizontalpanoramas ist, geht abermals aus einem Selbstzeugnis auf S. 496 bis 498 der Erstausgabe der "Voyages» hervor: "L'idée de cette espèce de dessin me vint sur le Buet même en 1776. Lorsque j'eus achevé la description des objets infiniment variées que j'avois sous les 


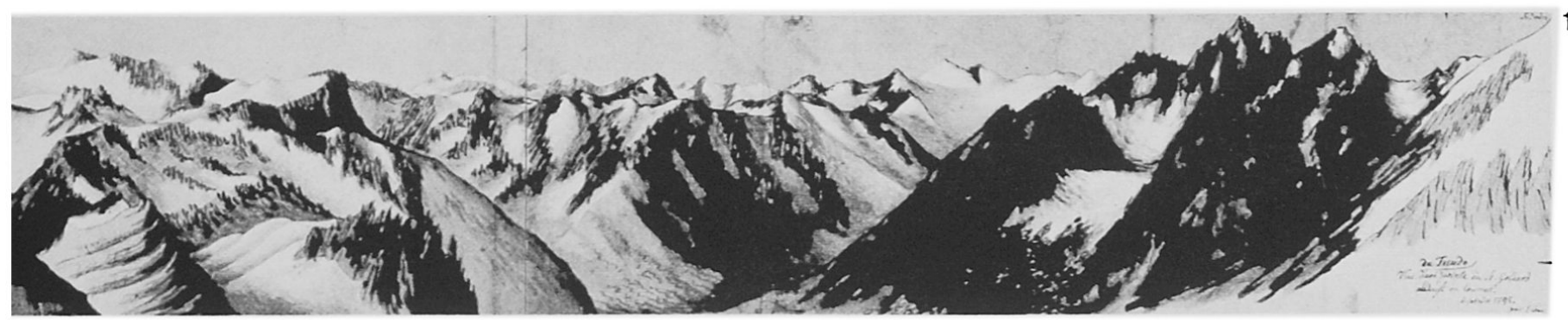

yeux, je vis clairement qu'il me seroit impossible d'en donner à mes Lecteurs une idée un peu nette sans y joindre des dessins. Mais en employant des vues ordinaires, il en auroit fallu un grand nombre; \& plus elles auroient été nombreuses, moins elles auroient rendu l'ensemble \& l'enchaînement de toutes ces montagnes, comme on les voit dans la Nature. Il faut dans le Dessinateur un singulier effort d'attention, \& une application difficile des règles de la perspective, pour projetter sur des plans verticaux \& sur des lignes droites, des objets qu'il voit réellement sur les circonférences \& dans l'intérieur d'un nombre de cercles dont son œil est le centre. Et il faut les mêmes efforts de la part du Lecteur, pour faire l'inverse du travail du Peintre, en se figurant sur des circonférences de cercle, ce que le dessin lui présente, en ligne droite.»

Saussure bestätigt auf diese Weise, daß das VertikalRundpanorama zwar möglich, aber äußerst schwierig anzufertigen wäre, d.h. daß es ein solches zu seiner Zeit noch nicht gab, obwohl es ihm theoretisch bereits vorschwebte. Damit ist Saussure nicht nur der Erfinder des Horizontaltyps, sondern auch der Theoretiker des Vertikaltyps des Rundpanoramas und unmittelbarer Vorläufer Eschers. Was die Erfindung des horizontalen Panoramatyps betrifft, konnte Saussure allerdings nicht ahnen, daß er bereits erfunden war: 1529 zeichnete ein «berühmter Maler", vermutlich Sebald Beham, vom Stephansturm in Wien die Belagerung der Stadt durch die Türken als Horizontalpanorama, das von dem Nürnberger Verleger Niklas Meldemann gedruckt und herausgebracht wurde. Derartiger Horizontalpanoramen gab es im Nürnberg des 16. Jahrhunderts mehr.

Saussure führt dann weiter aus: "Au contraire, suivant la méthode que j'ai employée, le Dessinateur peint ses objets exactement comme il les voit, en tournant son papier à mesure qu'il se tourne luimême, et ceux qui d'après son ouvrage, veulent se former une idée des objets qu'il a dessinés, n'ont qu'à se figurer qu'ils sont placés au centre du dessin, agrandir par l'imagination ce qu'ils voyent au dessus de ce centre, \& faire, en tournant le dessin, la revue de toutes ses parties. Ils voyent ainsi successivement tous les objets liés entr'eux, \& absolument tels qu'ils se présentent à un Observateur situé sur le sommet de la montagne.) Das ist eine fertige
Begründung der späteren Anwendung dieses Panoramatyps und eine Gebrauchsanweisung. Im weiteren gibt Saussure exakte Hinweise, wie ein solches Panorama zu zeichnen sei, und erklärt, sein Zeichner M.-Th. Bourrit, dem er seine Idee 1776 mitgeteilt habe, hätte sie begeistert aufgegriffen und sogleich auf dem Buet-Gipfel verwirklicht. Es ist auch Bourrit gewesen, dem wir dank Saussure präzise panoramatische Ansichten - also nicht Panoramen, sondern gewissermaßen Breitwandaufnahmen - des Hochgebirges verdanken, wie sie Escher wenig später zum Haupttypus der Gebirgsansicht machte.

Auch die dritte (oder chronologisch erste) schweizerische Erstleistung auf dem Gebiet des Panoramas gehört einem Welschschweizer. Die Daten über diesen genialen Feuerkopf sind ebenso bekannt wie sein Werk: Der Physiker und Mathematiker JACQUESBARTHELÉMY MICHELI DU CREST $(1699-1766)^{11)}$ zeichnete als Staatsgefangener auf der Festung Aarburg, in der er seit 1749 unschuldig eingekerkert war ${ }^{12)}$, im Jahre 1754 mit den primitivsten Vermessungsbehelfen wie z. B. einer zweckentfremdeten Dachrinne das von seinem Gefängnis aus sichtbare Alpenpanorama und ließ 1755 durch Vermittlung A. v. Hallers und Baviers, eines Freundes in Basel, seinen «Prospect géométrique des Montagnes neigées, dittes Gletscher, telles qu'on les découvre en tems favorable depuis le château d'Arbourg. 1755.» als Radierung T.C. Lotters in Augsburg herausgeben. Im Kommentar ${ }^{13)}$ erklärt Micheli, kein Zeichner zu sein und seine Vermessungsergebnisse nur notgedrungen zeichnerisch festgehalten zu haben. Dessenungeachtet ist sein Alpenpanorama das erste moderne Gebirgspanorama überhaupt. Seine einem Brief an A. v. Haller beigefügte vorbereitete Skizze von 1754 befindet sich in der Zentralbibliothek Luzern, wo J. H. Graf sie 1891 unter den Zeichnungen F.L. Pfyffers von Wyher erkannte und bestimmte ${ }^{14)}$. Der «Prospect géométrique» enthält z. T. fehlerhafte Angaben, die schon F. L. Pfyffer, der Schöpfer der ersten Reliefs der Zentralschweiz (1769), nachwies, um Micheli jedoch in einem Schreiben an Saussure wieder zu entlasten: "Son opération était belle pour un homme renfermé dans un bastion et qui ne pouvait travailler que d'un seul point. La faute rétombe plus sur ceux qui l'ont trompé sur les noms» ${ }^{15}$ ). Welch eine Leistung dieses erste Gebirgspanorama zu seiner Zeit war, zeigt der 
Abb. 1: "Vue du Fieudo, une Pointe du St. Gothard à l'ouest du Couvent, dessiné en 1792, par Escher." Erstes GebirgsRundpanorama der Welt. Aquarellierte Federzeichnung über Stiftvorzeichnung. Graph. Sammlung der ETH Zürich. Ausschnitt.

Abb. 2: M.-Th. Bourrit nach Anweisung H. B. de Saussures: Rundsicht vom Buet. Erstes Horizontalpanorama der Welt. Radierung 1776, im 1. Band der "Voyages dans les Alpes" von H. B. de Saussure.

2

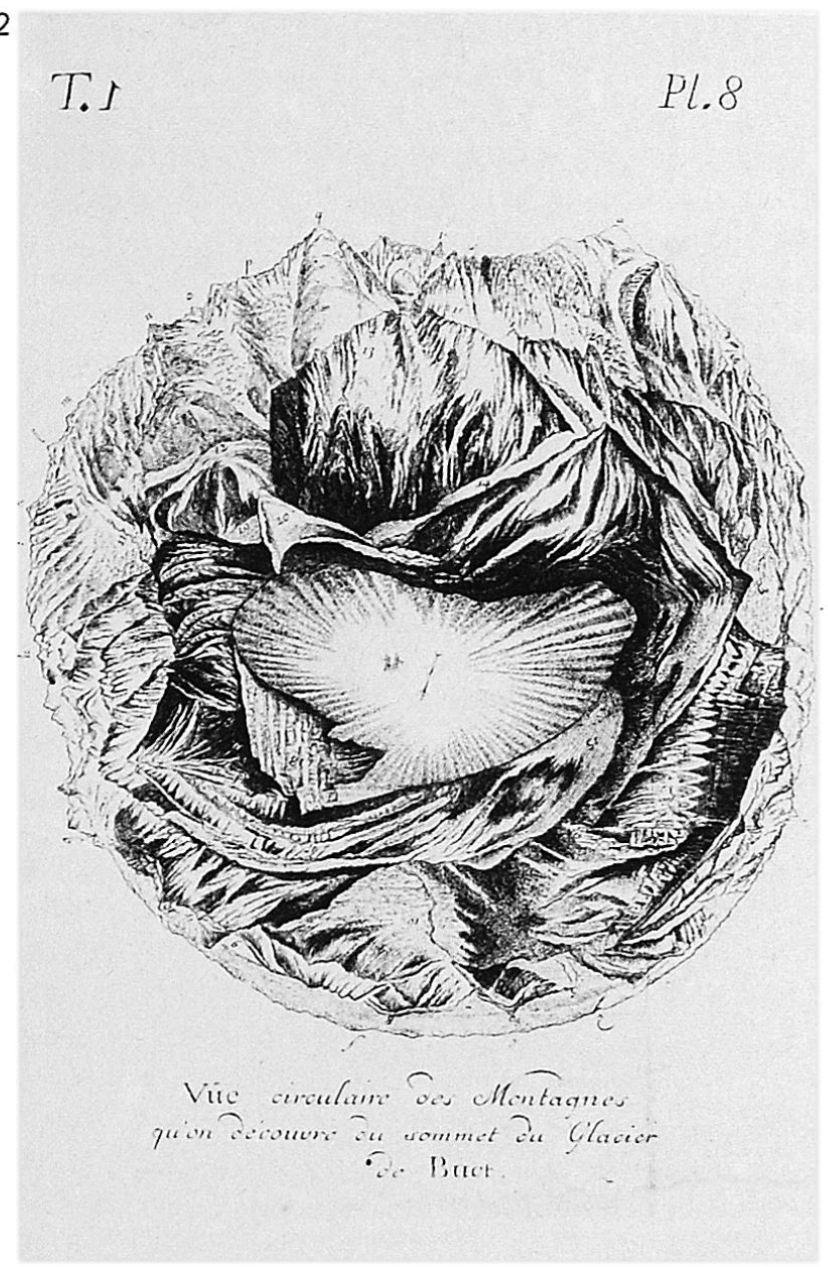

Abb. 3: J. B. Micheli du Crest: Die Alpenkette von der Aarburg aus. Entwurf 1754 (Zentralbibliothek Luzern), Radierung 1755 von T. C. Lotter in Augsburg.

Abb. 4: Gabriel Walser, Die Alpsteinkette,-1760. Aquarellierte Federzeichnung. Kartensammlung der Zentralbibliothek Zürich.

Abb. 5: Hans Conrad Escher in der Zeit zwischen 1802 und 1807, das einzige Porträt aus seinen besten Mannesjahren, gezeichnet von Hans Jakob Oeri (Zentralbibliothek Zürich)

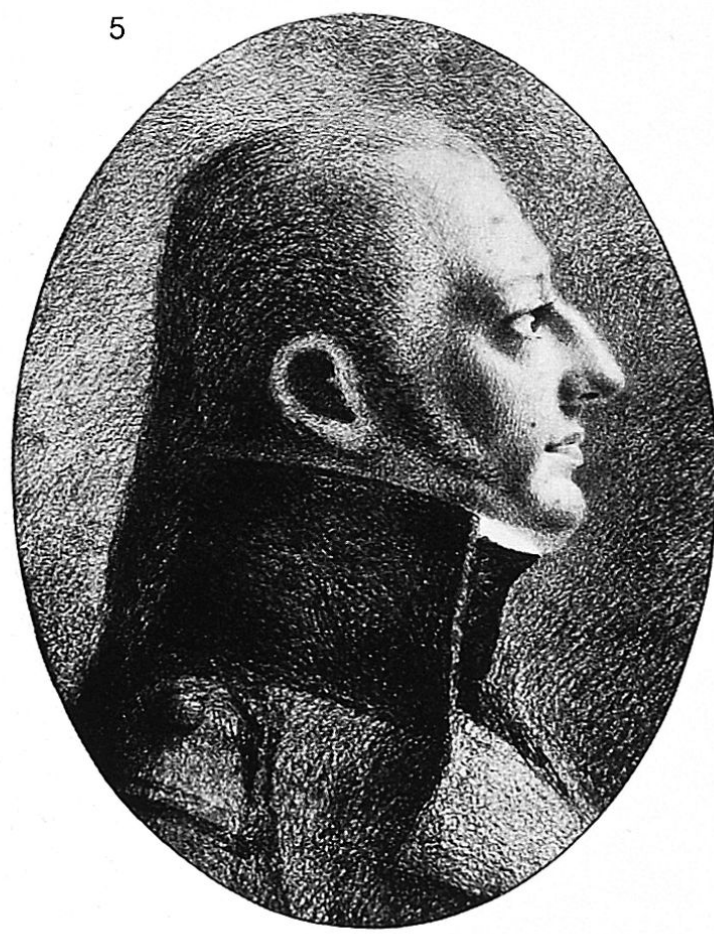

4 s.

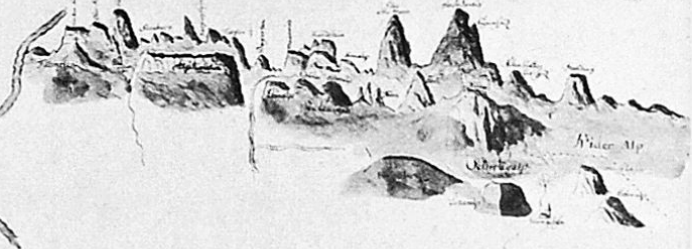

3

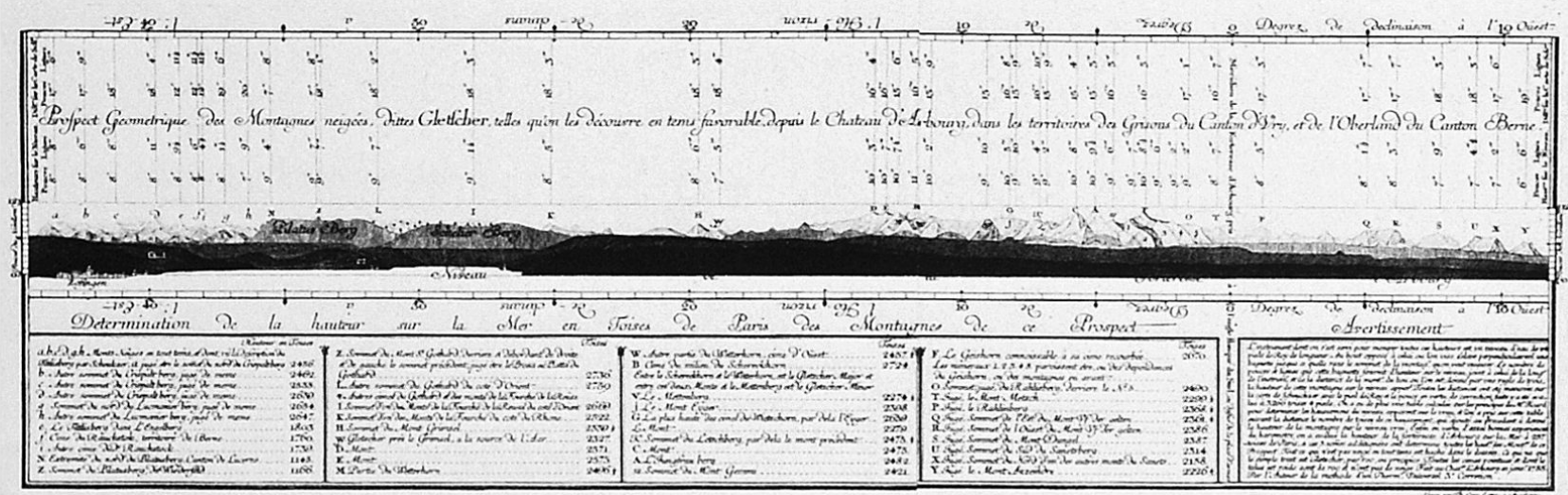


Vergleich mit dem Versuch GABRIEL WALSERS (1740 und 1760), die Alpsteinkette «wirklichkeitsgerecht» abzukonterfeien ${ }^{16)}$. Walsers mittelalterlich anmutende Bergformen sind noch weitgehend der schematischen Darstellungsweise verpflichtet und die Individualisierung bahnt sich erst ganz allmählich an. Michelis Arbeit hingegen gehört voll und ganz der neuen Zeit, was auch dem Fortschrittsgefälle zwischen der West- und der Ostschweiz um die Mitte des 18. Jahrhunderts entspricht.

Eine Übersicht der Literatur zum schweizerischen Panoramenschaffen wäre sehr kurz. BERNHARD STUDER widmet ihm in seiner Geschichte der physischen Geographie der Schweiz (Bern-Zürich 1865) einige wenige Seiten. Er ist der einzige, der seinen Saussure gut gelesen zu haben scheint, denn er erkennt das Buet-Panorama, wenn auch unter Vorbehalt, als Erstleistung an: «Der erste Versuch topographischer Rundansichten scheint von de Saussure, 1776 , ausgegangen und noch im gleichen Jahre durch Bourrit ausgeführt worden zu sein. So entstand das kleine Panorama des Buet im 1. Bande von Saussures Reisen.» Die Autoren der Folgezeit haben das anscheinend wieder vergessen. Auch RUDOLF woLF erwähnt es auf den 2 $1 \frac{2}{2}$ Druckseiten (115-117), die er in seiner Geschichte der Vermessungen in der Schweiz (1879) dem Panorama widmet, nicht. J. H. GRAF verzeichnet 1892 in einem Aufsatz von nur zwei Druckseiten ${ }^{17)}$ lediglich die Erstleistung Michelis, obwohl er auch Saussures Buet-Panorama anführt. Escher ist ihm nur als Theoretiker bekannt. Dafür nennt er Sigmund (fälschlich Samuel) Gottlieb Studers "Chaine des Alpes vue depuis les environs de Berne», 1788 von Dunker radiert, das älteste formenrichtige Panorama der Schweiz, was Michelis unter so schwierigen Bedingungen vollbrachte Leistung allerdings nicht schmälern kann, sowie einige weitere Vorleistungen zu Eschers großem Werk, das er natürlich nicht kennt. Später hat sich nur noch E. IMHOF in einer mehr als Causerie gedachten Übersicht ${ }^{18)}$ mit der Geschichte des schweizerischen Panoramas auseinandergesetzt, ohne indessen ihre Frühzeit um neue Erkenntnisse zu bereichern. Daß bisher nur Michelis große Leistung gewürdigt wurde, ist übrigens verständlich; diejenige Saussures mußte übersehen werden, solange die Rolle Eschers unbekannt war.

Wenn wir die Vedutistik des 18. Jahrhunderts auf
Panoramatisches hin untersuchen und von da aus die Entwicklung über die beiden Zweige Stadt- und Landschaftspanorama bis zu den spätmittelalterlichen Anfängen zurückverfolgen, nimmt eine Frühgeschichte des Panoramas Gestalt an, die bisher noch nicht geschrieben ist ${ }^{19)}$. Der Verfasser mußte diese zeitraubende, aber auch in seinem eigenen kunstgeschichtlichen Bereich ungemein ergiebige Arbeit in Angriff nehmen, um Eschers, Saussures und Michelis Leistung gerecht zu Die drei Marksteine werden ${ }^{20}$.

$\begin{array}{ll}1754 \text { Micheli du Crest } & \begin{array}{l}\text { erstes wissenschaftliches } \\ \text { Alpenvertikalpanorama }\end{array} \\ 1776 \text { Saussure } & \begin{array}{l}\text { erstes wissenschaftliches } \\ \text { Horizontalpanorama }\end{array} \\ & \text { erstes wissenschaftliches } \\ & \text { Vertikal-Vollrundpano- } \\ & \text { rama }\end{array}$

sind keineswegs zufällig schweizerische Erstleistungen, so wie die Qualität der schweizerischen Kartographie kein Zufall ist. Einem kleinen Volk, das sich mit dem Gebirge auseinandersetzen mußte, um zu überleben, sind diese Leistungen nicht geschenkt worden. Doch es hätte sich sie ohne das Rüstzeug und die neue Sicht, die mit dem Fortschritt aus Frankreich kamen, kaum erarbeitet. Nicht nur zufällig ist auch Escher ein Repräsentant des neuen freiheitlichen Geistes.

\section{Anmerkungen}

1) Ansichten und Panoramen der Schweiz 1780 bis 1822. Zum Lebenswerk H. C. Eschers von der Linth. Ausstellung im Predigerchor der Zentralbibliothek Zürich 9. 3.-5. 5. 1973, eröffnet zum 150. Todestag Eschers.

2) Den Text des erteilten Patents, aus dem diese Tatsache ersichtlich ist, bringt $\mathrm{H}$. Buddemeier im Dokumentationsteil seines Buches «Panorama, Diorama, Photographie - Ausbreitung und Wirkung neuer Medien im 19. Jahrhundert» (Reihe Theorie und Geschichte der Literatur und der schönen Künste), München 1970, zum Abdruck (S. 163). Damit ist die Ansicht G. Peyers auf S. 188 seiner Geschichte der Reisen in der Schweiz, Basel 1885, daß der Panoramenzeichner Hein- 


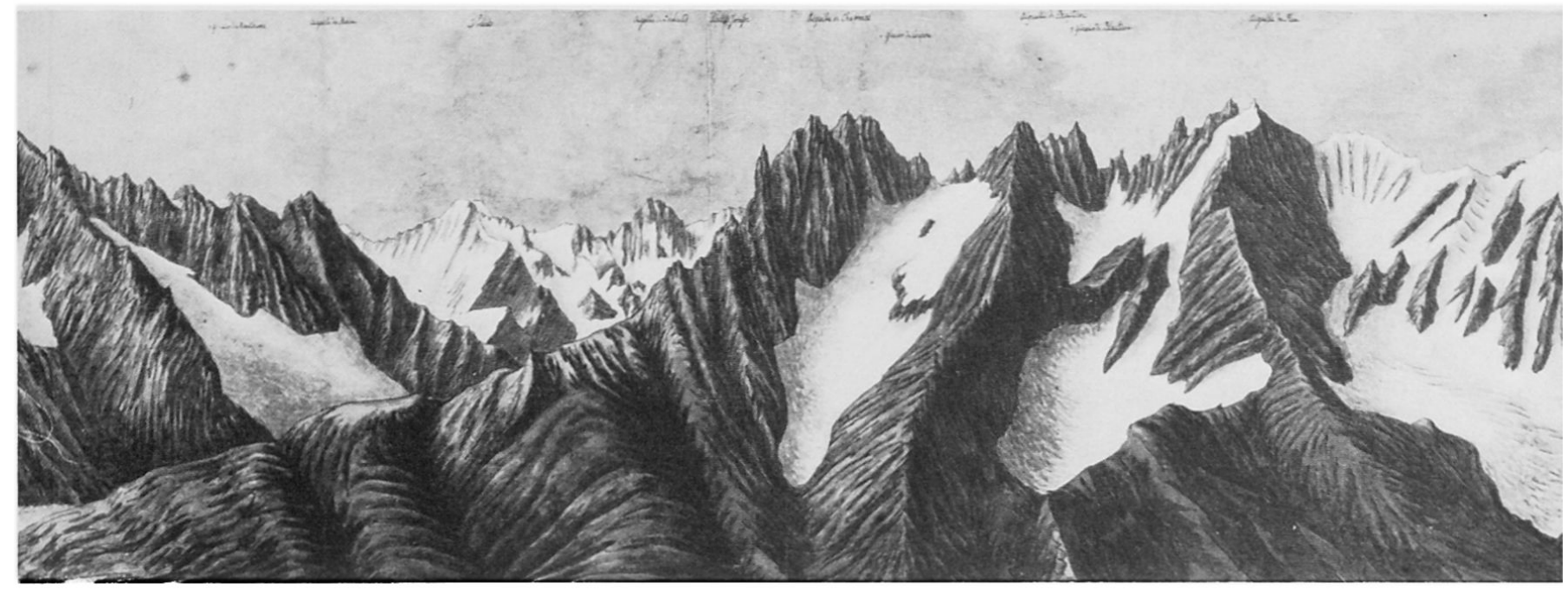

rich Keller «recht eigentlich den Namen ,Panorama' aufgebracht» habe, widerlegt.

3) J. A. Breysig, Skizzen, Gedanken-Umrisse; Entwürfe die bildende Kunst betreffend. Magdeburg 1798-1801.

4) Über J.H. Breysig s. Michael Hampe, Johann Adam Breysig, Porträt eines Theatermalers. Neue Zürcher Zeitung 4. 2. 1973, S. 52.

5) Taschenbuch für die gesamte Mineralogie mit Hinsicht auf die neuesten Entdeckungen, herausgegeben von Carl Caesar Leonhard. Franfurt a. M. 1812, S. 392-394.

6) Für diesen Hinweis danke ich B. Weber, der die Tatsache in seinen Erläuterungen zum erwähnten Zurlaubenschen Werk im Bildband «Berge und Städte der alten Schweiz», Basel 1973, auf S. 19 aufzeigt.

7) Warum diese Aufgabe gerade von schweizerischen Panoramenzeichnern gelöst worden ist, geht aus Johannes Firschaufs Aufsatz «Das Panorama als Hilfsmittel der Geographie» in Das Ausland, Wochenschrift für Erd- und Völkerkunde, Stuttgart 4.6.1892, hervor, wo es eingangs heißt: «Ist ... ein Hochgebirge kartographisch darzustellen, so ist es unmöglich, aus der Horizontalprojektion... sich ein klares Bild über die Form der Zacken und Kämme usw. zu machen; dann muß die eine (Horizontal-)Projektion ergänzt werden durch eine zweite - am besten auf eine Vertikalebene.» Die Schweiz, die sich für ihre
Eigenständigkeit zu wehren hatte (auch Escher hat eine Reihe militärisch-geographischer Gutachten ausgearbeitet), war diesbezüglich in einer ganz anderen Lage als beispielsweise Österreich, dessen Alpengebiet nie ein Réduit gewesen ist.

8) Die Publikation der Escher-Zeichnungen soll die vergänglichen, weil trotz größtmöglicher Schonung unter Lichteinwirkung verblassenden Farben der Nachwelt überliefern und zugleich die Originale allzustarker Beanspruchung entziehen helfen. Zur Herausgabe haben sich die Zürcher Verlage Atlantis und Orell Füßli zusammengefunden; im ersteren erscheint der Ansichtenband mit etwa 200 meist originalgroßen farbigen Reproduktionen, im letzteren eine Kassette mit 26 auf Syntosil gedruckten Panoramen in Faksimile und Originalformat sowie ein Werkverzeichnis.

9) s. Joh. Jak. Hottinger, Hans Conrad Escher von der Linth, Charakterbild eines Republikaners, Zürich 1852, S. 22.

10) ebenda, S. 24.

11) Über Micheli du Crest s. vor allem: R. Wolf, Geschichte der Vermessungen in der Schweiz, Zürich 1879, S. 107-109; - J. H. Graf, Leben und Wirken des Physikers und Geodäten J. B. Micheli du Crest, Bern 1890, Separatdruck aus Graf, Geschichte der Mathematik III, 2; - L. Seylaz, Micheli du Crest et les Alpes, in: Die Alpen, Jg. 29, Bern 1953.

12) Aus einem Schreiben Michelis vom 24.11. 1751 


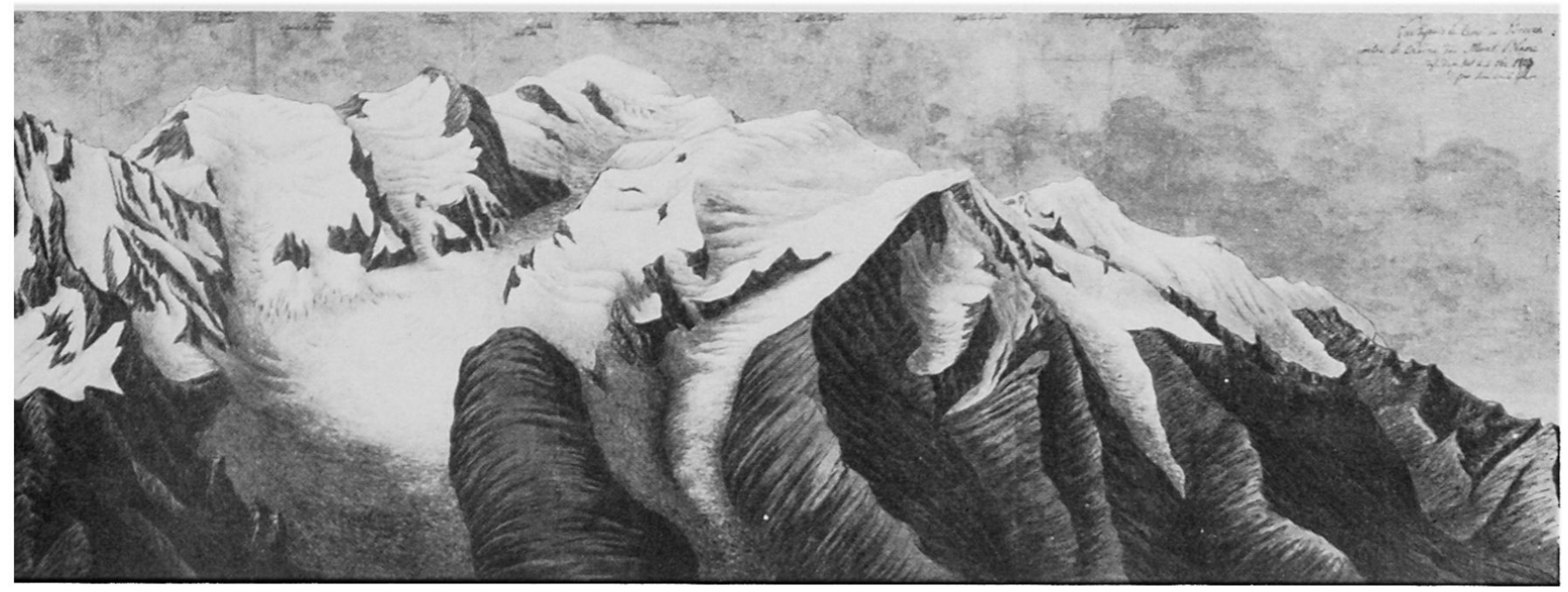

aus der Festung Aarburg an seinen Freund Bavier in Basel: "Je suis logé comme un chien, et de plus dans un souterrain très malsain, où je suis très souvent attaqué par des opressions de poitrine, et où je n'ai d'ailleurs pas la liberté de pouvoir me plaindre.»

13) Mémoire pour l'Explication du Prospect des Montagnes neigées que l'on voit du Château d'Arbourg. Fait au Château d'Arbourg le 10 Octobre 1754. Revu \& corrigé le 2 Juin 1755. Par l'Auteur de la Méthode d'un Thermomêtre Universel.

14) J. H. Graf, Das Originalpanorama eines Theils der Unterwaldner und Berner Alpen von J. B. Micheli du Crest, Jahrbuch des SAC 1892, S. 245 bis 252, Abb. im Beilagenteil.

15) Zit. nach R. Wolf (Anm. 11).

16) a) Prospekt des Alpsteins von Gabriel Walser, 1740 (Ausschnitt aus seiner Appenzeller Karte in Bischofsbergers Appenzellerchronik), Abb. in: Leo Weisz, Die Schweiz auf alten Karten, Zürich (2. Aufl.) 1969, Abb. 202 auf S. 204. - b) Ansicht der Alpsteinkette von Gabriel Walser, aquarellierte Federzeichnung, 1760, Kartensammlung der Zentralbibliothek Zürich. Vermutlich Entwurf zum entsprechenden Prospekt in Walsers Appenzeller Karte von 1768. Abb. in Turicum, Vierteljahresschrift für Kultur, Wissenschaft und Wirtschaft, Zürich, Winter 1973, S. 19. Der Verfasser des Beitrags H.P. Höhener bezeichnet diese Zeichnung allerdings zu Unrecht als das erste schweizerische Panorama, offenbar unter dem Einfluß von Leo Weisz (Anm. 16), der den Alpsteinprospekt sub a. sehr vage als das erste Panorama der Schweiz bezeichnet (S. 200), was angesichts der vielen hervorragenden Arbeiten schweizerischer Landschaftszeichner schon des 17. Jahrhunderts, vor allem Conrad Meyers, dahinfällt.

17) J. H. Graf, Die Entwicklung der schweizerischen Panoramenkunst, in: Das Ausland, Wochenschrift für Erd- und Völkerkunde, Stuttgart 1892, Jg. 65, Nr. 19, S. 292-294.

18) E. Imhof, Zürcher Kartenkünstler und Panoramazeichner, in: Zürich Vorhof der Alpen, Festgabe zum 100jährigen Bestehen der Sektion UTO SAC, Zürich 1963.

19) H. Buddemeier bemerkt in der Einleitung zu seinem erwähnten Buch (Anm. 2): "Auf Arbeiten, die die genannten Erfindungen im Zusammenhang untersuchen und sich dabei auf zeitgenössische Dokumente stützen, konnte nicht zurückgegriffen werden.» Wolfgang Krönig schreibt in seiner Rezension von Max Schefold, Alte Ansichten aus Baden, Weissenhorn/Ulm 1971, in der Kunstchronik, Heft 1, München 1973: «Zumal zum Panorama und seiner Vorgeschichte im 18. Jahrhundert wäre eine eigene Untersuchung längst fällig.»

20) Ein erster Abriß wird die in Anm. 7 erwähnte Kassette der Escher-Panoramen begleiten. 